\title{
Bright Room-Temperature Phosphorescence from Mixed Mothballs Enabling Specific Identification of the Illegal Component
}

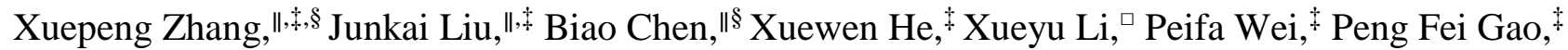 \\ Guoqing Zhang, ${ }^{\S}$ Jacky W. Y. Lam,${ }^{\ddagger}$ and Ben Zhong Tang $*,+, \dot{\dagger}$, I
}

'Shenzhen Institute of Aggregate Science and Technology, School of Science and Engineering, The Chinese University of
Hong Kong, Shenzhen, 2001 Longxiang Boulevard, Longgang District, Shenzhen City, Guangdong 518172, China
tDepartment of Chemistry, Hong Kong Branch of Chinese National Engineering Research Centre for Tissue Restoration and
Reconstruction, Division of Life Science and State Key Laboratory of Molecular Neuroscience, Institute for Advanced Study
and Institute of Molecular Functional Materials, The Hong Kong University of Science and Technology, Clear Water Bay,
Kowloon 999077, Hong Kong, China

${ }^{\S}$ Hefei National Laboratory for Physical Sciences at the Microscale, University of Science and Technology of China, Hefei 230026, China

${ }^{\square}$ Global Station for Soft Matter, Global Institution for Collaborative Research and Education, Hokkaido University, 001-0021 Sapporo, Japan

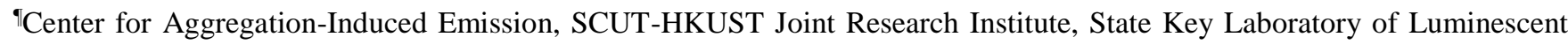
Materials and Devices, South China University of Technology, Guangzhou 510640, China

\begin{abstract}
Ultralong organic room-temperature phosphorescence (RTP) with high brightness was rarely achieved to date despite their huge potential in various applications such as lighting, sensing, anti-counterfeiting and imaging. Herein, by exploiting $\pi-\pi^{*}$ nature of the lowest excited triplet state of naphthalene (NL, the traditional active ingredient for mothball) and intersystem crossingpromoting factors from 1,4-dichlorobenzene (DCB, a safer alternative to NL as mothball), we report a simple and novel guest/host system, namely NL/DCB, that could produce strong green RTP with quantum yield $>20 \%$ and lifetime $>0.76 \mathrm{~s}$ (afterglow duration $>10 \mathrm{~s}$ ) at ambient conditions. The RTP performance with simultaneous high efficiency and ultralong lifetime is superior to that of most purely organic (metal-free) RTP materials reported so far. Control experiments with different hosts and first-principle theoretical calculations revealed that the robust RTP behavior in the unique NL/DCB system was mainly attributed to a combination of clusterexciton formation and external heavy atom effect. Meanwhile, the remarkable "turn-on" type RTP to naked eyes allows fast and specific detection of illegal NL mothball using DCB as a sensor, which is valuable in household as well as industrial applications.
\end{abstract}

\section{INTRODUCTION}

Naphthalene (NL) mothballs have been used as insect repellents and deodorants worldwide for a long period of time. ${ }^{1}$ Meanwhile, a variety of important chemicals are synthesized using NL as precursor in industry. ${ }^{2}$ However, application of the products always leads to high indoor concentration of NL vapor due to their rapid sublimation at room temperature, which is detrimental to human beings considering its severe toxicity (including capacity to cause hemolysis and organ injury), carcinogenicity and high flammability. ${ }^{3-7}$ In addition to inhalation of NL vapor, children and old people tend to swallow the whole NL balls by thinking of it as sugar, which could be life-threatening. ${ }^{3}$ The World Health Organization and many countries have designated NL as illegal component for mothballs. However, this has not yet contained the spread of NL mothballs which are often mislabeled as natural camphor by illicit manufacturers. It is challenging for law enforcers and consumers to distinguish NL from camphor (1,7,7-trimethylbicyclo [2.2.1] heptan-2-one) by routine density method since additives can be incorporated to make NL balls also lighter than water. Furthermore, NL mothballs have similar appearance and odor to camphor balls. ${ }^{8}$ Therefore, a specific method that is exclusive for NL identification is in urgent demand.
Luminescence spectroscopy is broadly employed in various disciplines such as analytical chemistry and biosciences since it tells featured information about the electronic states of a given compound. Compared to steady-state luminescence spectroscopy, afterglow spectroscopy can filter interference from excitation light by time-resolved technique, allowing for higher signal-to-noise ratio. ${ }^{9-10}$ In addition, afterglow phenomenon is fairly intuitive and distinguishable to naked eyes. ${ }^{11-16}$ As a result, many ultralong afterglow materials (including RTP and charge separation-recombination ${ }^{13-14,16-17}$ systems) have been developed in recent years, which show potential applications in lighting, ${ }^{18}$ displaying, ${ }^{19}$ encryption, ${ }^{17,}$ 20-32 imaging $^{33-35}$ and advanced anti-counterfeiting. ${ }^{36-39}$ Currently, improving the efficiency or brightness of ultralong afterglow remains a central challenge, with very limited highly emissive cases (QY in order of magnitude of $10 \%$ or higher) reported to date using well-designed material structures such as cocrystals, ${ }^{40}$ assemblies ${ }^{41}$, hydrogenbonding ${ }^{31}$ and carbon dots in selected matrix ${ }^{38}$.

In respect of RTP emitter (the more versatile source for afterglow in organic system), both aromatic and non-aromatic phosphor has been developed with the former being more typical. ${ }^{42}$ As the simplest polycyclic aromatic hydrocarbon, NL, however, its phosphorescence property has been mainly investigated at 


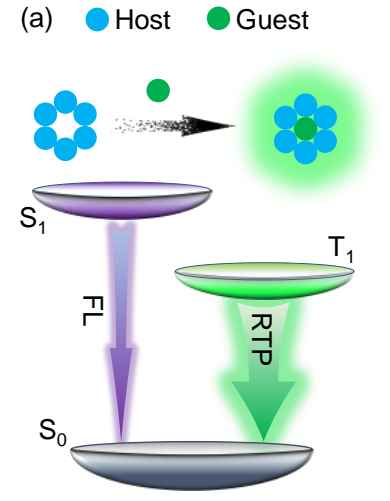

(b)

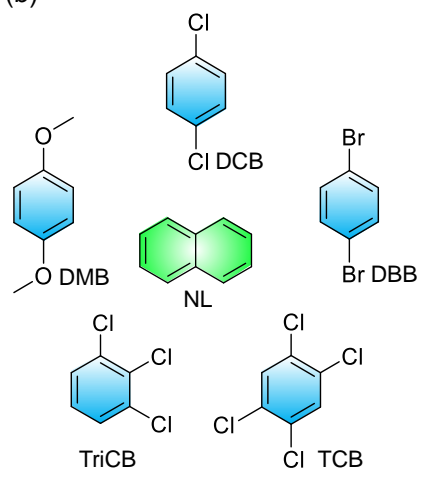

Figure 1. (a) Schematic illustration of the guest/host system showing ultralong room-temperature phosphorescence (RTP). (b) Effective hosts (blue) for promoting RTP of naphthalene. Abbreviations: NL for naphthalene, DMB for 1,4-dimethoxybenzene, DCB for 1,4dichlorobenzene, TriCB for 1,2,3-trichlorobenzene, TCB for 1,2,4,5-tetrachlorobenzene and DBB for 1,4-dibromobenzene.

cryogenic temperature by spectroscopists. ${ }^{43-45}$ Instead, RTP of NL seems to be observable with relatively low QY only in complex micellar or supra-molecular structure under stringent deoxygenation condition. ${ }^{46}$ This situation raises an important question: can NL exactly produce efficient ultralong phosphorescence that is useful in practical application at ambient temperature and atmosphere? In principle, the lowest excited triplet state $\left(\mathrm{T}_{1}\right)$ of NL is of pure $\pi-\pi^{*}$ nature and thus the spin-forbidden $\mathrm{T}_{1}-\mathrm{S}_{0}$ transition can lead to ultralong phosphorescence lifetime. However, weak spin-orbit coupling (SOC) and planar conformation of NL molecule (usually prone to aggregation-cause quenching, ACQ) make bulk NL difficult to show RTP. Thus, to achieve efficient RTP for NL, at least three requirements should be met: (1) the NL molecules is dispersed in a matrix to avoid bulk aggregation; ${ }^{47-49}$ (2) the matrix is rigid and dense enough at RT to minimize molecular-motion and oxygen quenching; ${ }^{11}$ (3) since NL lacks "one-center" $\mathrm{p}_{\mathrm{x}} \leftrightarrow \mathrm{p}_{\mathrm{y}}$ orbital transition that is required for flip or rephrase of electron spin, ${ }^{21}$ the host molecule need to be ISC-promoting.

Accordingly, on one hand, halogen-containing host is expected to be effective as a result of external heavy atom effect. ${ }^{50-}$

${ }^{51}$ On the other hand, should the host molecules form cluster excitons with NL, ISC will be further boosted. ${ }^{52}$ To this end, the host is preferred to have excited-state energy lying closely above that of the guest. Meanwhile, electron-donating/withdrawing abilities of the host and the guest should not differ too much. ${ }^{52-56}$ Coincidentally, DCB, a safer and $2^{\text {nd }}$-generation mothball which was suggested to replace NL, fulfills all the necessary criteria as a host to activate the RTP of NL. As anticipated, strikingly bright and ultralong afterglow was realized in the "simple" NL/DCB guest/host system at ambient conditions. In the current work, we systematically investigated the mechanism of this RTP phenomenon and demonstrated its potential to serve as a powerful optical tool in sensing applications.

\section{RESULTS AND DISCUSSION}

Luminescent properties of the guest/host samples. To gain a detailed understanding about the influence of host on the photophysical properties of NL, we selected halogen-free (DMB) and a series of halogen-containing (TriCB, TCB, DBB) benzene derivatives as control in addition to DCB (Figure 1). All the hosts
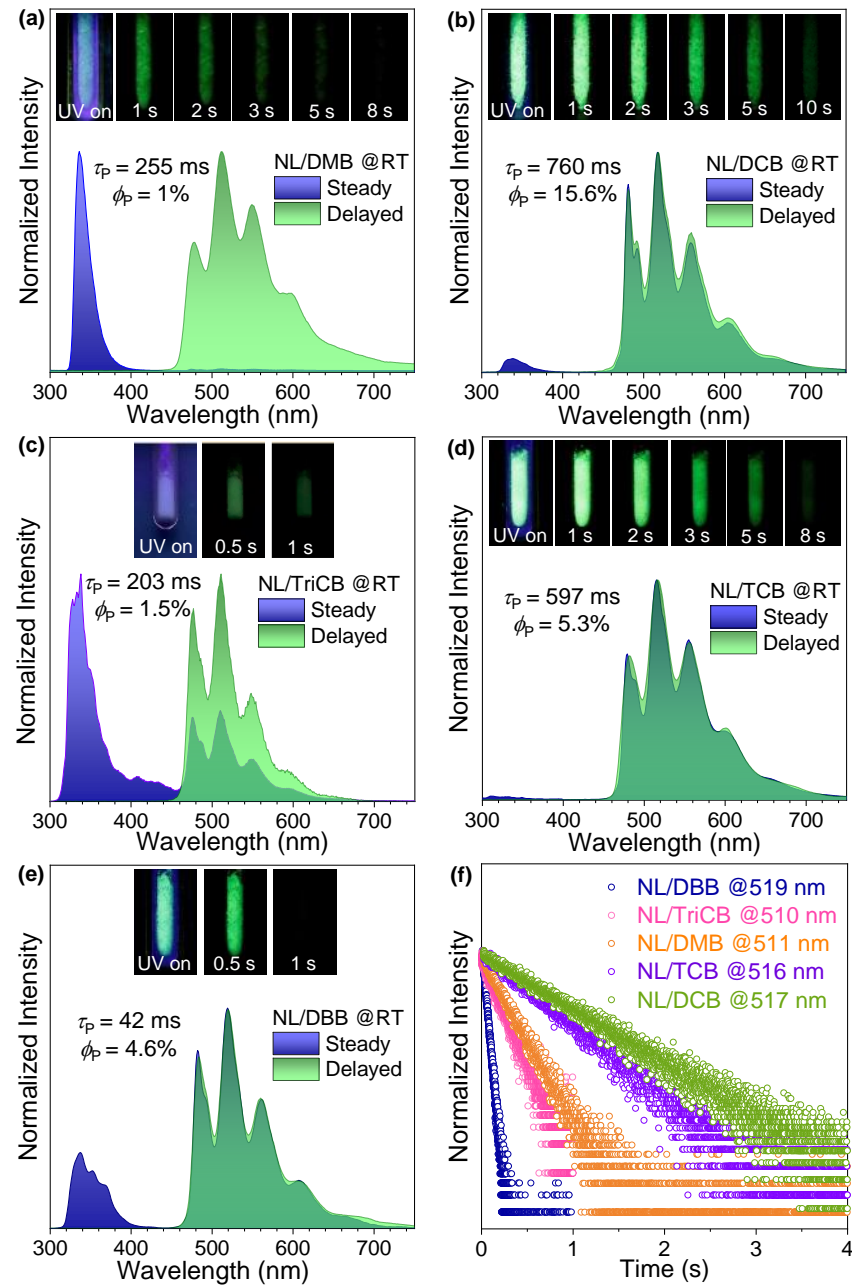

Figure 2. (a) (e) Steady-state and delayed $(\Delta t=50 \mathrm{~ms})$ PL spectra of NL/host system (NL/DMB, NL/DCB, NL/TriCB, NL/TCB and $\mathrm{NL} / \mathrm{DBB}$, respectively) at ambient conditions. Insert: Photos of NL/host when 254-nm UV light was on and off at ambient conditions; RTP lifetime ( $\tau$ P) and QY ( $\phi \mathrm{p})$ values were also shown. (f) Time-resolved decay curves of NL/host samples at maximum RTP emission. The samples were fabricated via melt-casting with starting mass ratio of NL/host $=1 / 100$, respectively. $\left(\lambda_{\mathrm{ex}}=280 \mathrm{~nm}\right)$

and NL were purified with silica-gel chromatography using distilled solvent and their purity were verified by high-performance liquid chromatography prior to use. These single-component materials did show relatively weak fluorescence but no RTP (afterglow) was observed at all. Specifically, the steady-state PL spectrum of $\mathrm{NL}$ in $\mathrm{CH}_{2} \mathrm{Cl}_{2}$ shows a fluorescence band $\left(\phi_{\mathrm{FL}}=\right.$ $14 \%$ ) centered at $327 \mathrm{~nm}$ with several vibrational peaks (337 and $341 \mathrm{~nm}$ ). In comparison, the NL solid exhibits major emission peak centered at $340 \mathrm{~nm}$ with decreased QY $\left(\phi_{\mathrm{FL}}=9 \%\right)$ and slightly blurred vibrational patterns. As for the host solids, DMB show emission peak at $332 \mathrm{~nm}\left(\phi_{\mathrm{FL}}=3 \%\right)$ while halogenated hosts all exhibited very faint fluorescence in the UV region with QY $<1 \%$ (Figure S1).

Guest/host samples were then fabricated via melt-casting at starting mass ratio of $1 / 100$ between NL and different hosts, respectively. For NL/DMB (Figure 2a), weak blue luminescence was observed under 254-nm light irradiation and a green afterglow could last for $\sim 8 \mathrm{~s}$ at ambient conditions following the cease of UV irradiation. Consistently, steady-state photoluminescence (PL) spectrum of NL/DMB shows major fluorescence 
band centered at $\sim 336 \mathrm{~nm}\left(\tau_{\mathrm{FL}}=3.4 \mathrm{~ns}\right)$ and tails to the bluelight region. A weak phosphorescence band centered at $511 \mathrm{~nm}$ $\left(\tau_{\mathrm{P}}=255 \mathrm{~ms}\right)$ with vibrational fine structure is noticed when zoomed in (Figure S2), which is consistent with the delayed emission spectrum. The fluorescence band is attributed to a mixture of NL dispersed in DMB at molecular level, bulk DMB and bulk NL. While the phosphorescence band was assigned to triplet excited-state emission of NL dispersed in DMB at molecular level, which is verified by the phosphorescence spectrum of NL in 4:1 ethanol-methanol glass $\left(10^{-3} \mathrm{M}\right)$ at $77 \mathrm{~K}$ (Figure S3). The RTP of NL/DMB was so weak that the quantum yield was measured to be only $1 \%$. Even cooled to $77 \mathrm{~K}$, NL/DMB still show dominant fluorescence band over phosphorescence (Figure S4). The inefficient RTP of NL/DMB indicate that DMB mainly provides an inelastic matrix for NL molecule but did not enhance the ISC of NL evidently.

In comparison, NL/DCB, NL/TCB and NL/DBB all show much more intense green RTP emission to naked eyes under 254-nm UV light irradiation at ambient conditions, with that of NL/DCB being the brightest (Figure 2b, 2d and 2e). After UV excitation ceased, NL/DCB also exhibits the longest afterglow ( $11 \mathrm{~s})$, followed by NL/TCB ( 8 s). However, afterglow duration of NL/DBB is only $\sim 1 \mathrm{~s}$. Consistently, the RTP band of NL in these halogen-containing hosts dominates the steady-state PL spectrum, almost overlaps with the delayed emission spectrum. Additionally, RTP lifetimes of NL/DCB, NL/TCB and NL/DBB are measured to be $760 \mathrm{~ms}, 597 \mathrm{~ms}$ and $42 \mathrm{~ms}$, respectively, while RTP QYs of NL/DCB, NL/TCB and NL/DBB are $15.6 \%, 5.3 \%$ and $4.6 \%$, respectively $\left(\lambda_{\mathrm{ex}}=280 \mathrm{~nm}\right)$. Among the studied halogenated hosts, TriCB appears to be an exception since the RTP intensity of NL/TriCB $\left(\phi_{\mathrm{P}}=1.5 \%, \tau_{\mathrm{P}}=203 \mathrm{~ms}\right.$, duration $<2 \mathrm{~s}$ ) is comparable to that of NL/DMB. At this stage, it is noted that most of the investigated halogen-containing hosts promote the RTP efficiency of NL substantially compared to DMB. However, there is no quantitative relationship between the RTP properties and the external heavy atom effects. On one hand, from NL/DMB to NL/DCB to NL/DBB, the RTP QY increase (by a factor of $>15$ to 4.6 ) is much more than can be accounted for by an increase in atomic number in going from $\mathrm{O}$ to $\mathrm{Cl}$ to $\mathrm{Br}$ on the host substituent. On the other hand, from NL/DCB to NL/TriCB to NL/TCB, the NL RTP did not enhance with increasing number of $\mathrm{Cl}$ atoms on the host. The reality is that DCB (with moderate atomic and substituting number of halogen) could activate the NL RTP to the greatest extent. These results might be partially rationalized by the fact that SOC enhancement is determined by how close the NL $\pi$ electron can approach the halogen nucleus on the host molecule, not only atomic and substituting number of the halogens. Indeed, the heterogeneity and intricate molecular rearrangement in the guest/host system make it difficult to predict the distance between NL electron and halogen with precision when substituent on the host varies. ${ }^{57}$

Cluster-exciton mechanism for NL/DCB RTP. As shown in Figure 3a-b, the NL/DCB RTP band with characteristic vibrational patterns $(481,517,559$, and $605 \mathrm{~nm})$ could always appear without any shift under varying UV excitation from 250 to over $320 \mathrm{~nm}$. What changes with excitation wavelength is the intensity of the whole RTP band. These results indicate that there is always only one emitting state for the RTP band, i.e., the $T_{1}$ of NL molecule in the NL/DCB system, regardless of different excitations. The question now is what happened in the excited state before the exciton decays to $T_{1}$ of NL. As shown in Figure $3 \mathrm{c}$, the excitation spectrum of NL/DCB monitored at maximum RTP emission $(517 \mathrm{~nm})$ is almost as broad as that of NL in
$\mathrm{CH}_{2} \mathrm{Cl}_{2}$ solution from 240 to over $300 \mathrm{~nm}$. In the range of 240 290 nm, the excitation spectrum of bulk DCB also largely overlaps with that of $\mathrm{NL}$ in $\mathrm{CH}_{2} \mathrm{Cl}_{2}$ (Figure $3 \mathrm{c}$ ). Moreover, when excited by 260, 270, 280, 290 and $300 \mathrm{~nm}$ UV light respectively, the RTP QYs are all higher than $15 \%$ (Figure 3e), with QY being over $20 \%$ when excited by 260 and $290-n m$ UV light. Thus, in the process of producing strong RTP, both NL molecule and bulk DCB could be excited by the wide range of UV light. This makes it less reasonable to interpret the RTP as a consequence of energy transfer from the excited-state host to the guest. Furthermore, at guest concentrations from $10 \%, 5 \%, 1 \%$ to $0.1 \%$, NL/DCB always show strong green RTP to naked eyes. For concentration of $0.1 \%$ and $1 \%$, the RTP band dominates the steady-state PL spectra and the highest $\mathrm{I}_{\mathrm{P}} / \mathrm{I}_{\mathrm{F}}$ values (RTP intensity over fluorescence intensity) are achieved (Figure 3d, 3f). From $1 \%$ to $5 \%$ to $10 \%$, the NL fluorescence band $(\sim 337 \mathrm{~nm})$ gradually increases to surpass the RTP band. At a low content of $0.01 \%$, NL/DCB still show noticeable green RTP yet the emission intensity decreased heavily compared to that of $0.1 \%$. Moreover, only faint green afterglow was observed at $10 \mathrm{ppm}$
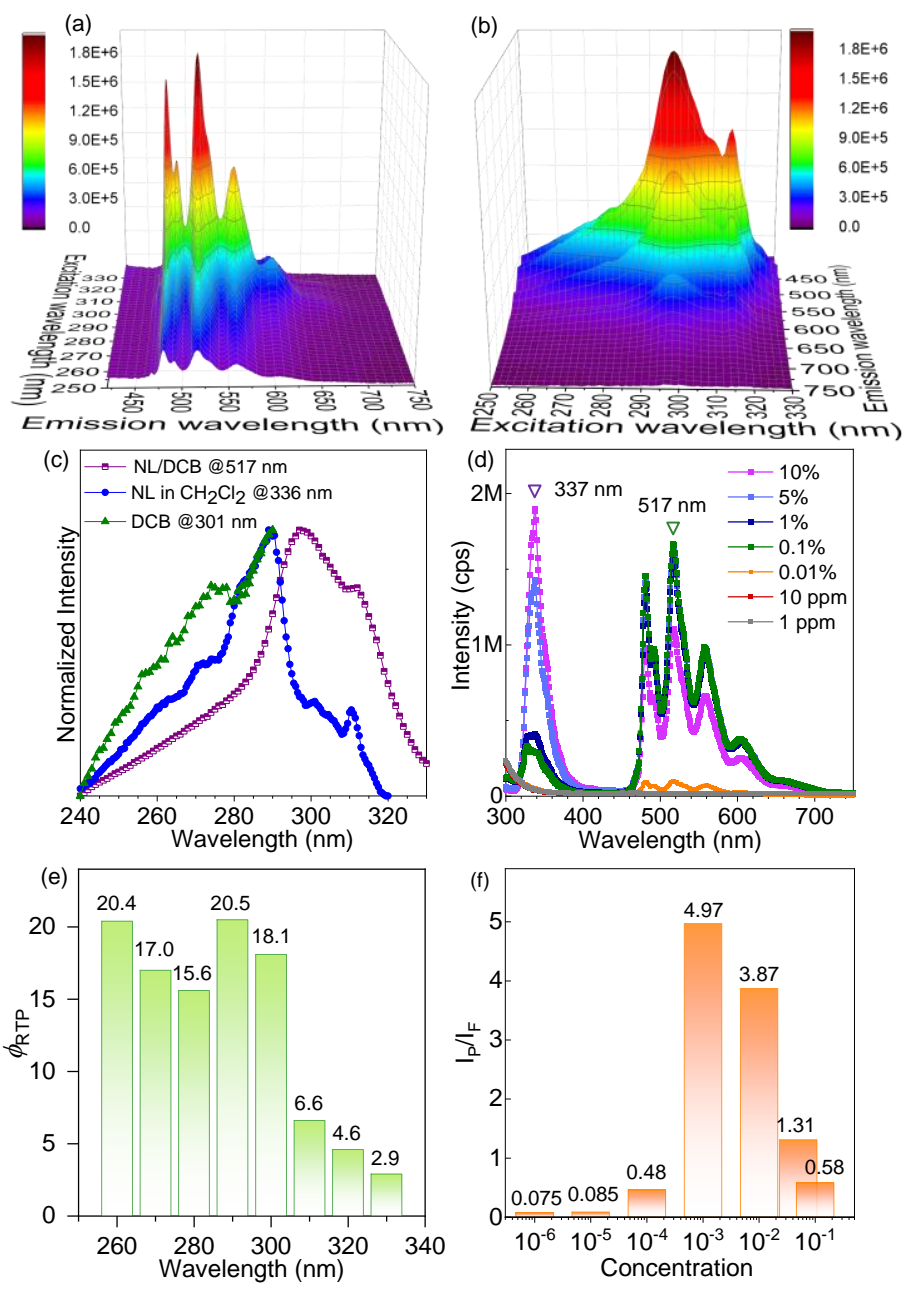

Figure 3. (a) (b) Excitation-phosphorescence mapping of NL/DCB at RT from view of emission and excitation, respectively. (c) Excitation spectra of NL/DCB monitored at $\lambda_{\mathrm{em}}=517$ $\mathrm{nm}, \mathrm{NL}$ in $\mathrm{CH}_{2} \mathrm{Cl}_{2}$ monitored at $\lambda_{\mathrm{em}}=336 \mathrm{~nm}$ and bulk DCB monitored at $\lambda_{\mathrm{em}}=301 \mathrm{~nm}$. (d) Steady-state PL spectra of NL/DCB with different starting guest/host mass ratios at RT ( $\lambda_{\text {ex }}$ $=290 \mathrm{~nm})$. (e) RTP $(450 \sim 750 \mathrm{~nm})$ QYs of NL/DCB under different excitation wavelengths. (f) Corresponding $\mathrm{I}_{\mathrm{P}} / \mathrm{I}_{\mathrm{F}}$ values (RTP intensity over fluorescence intensity) extracted from (d). 
(a)

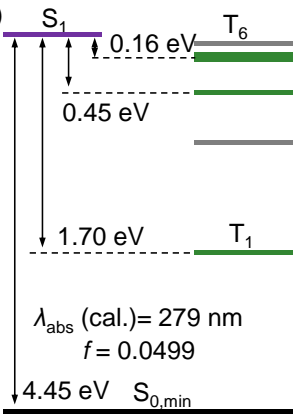

(b)

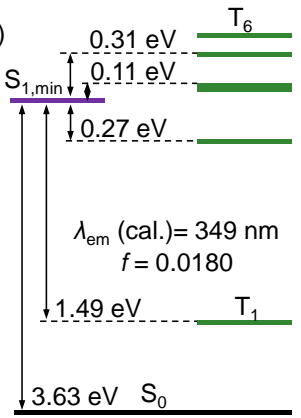

(c)

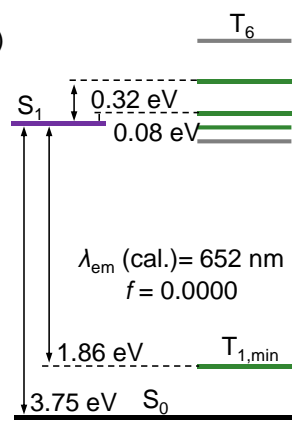

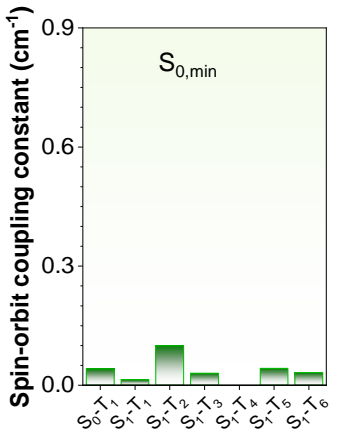
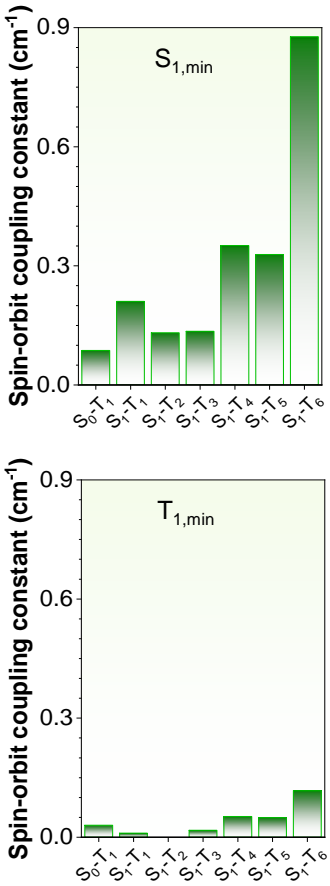
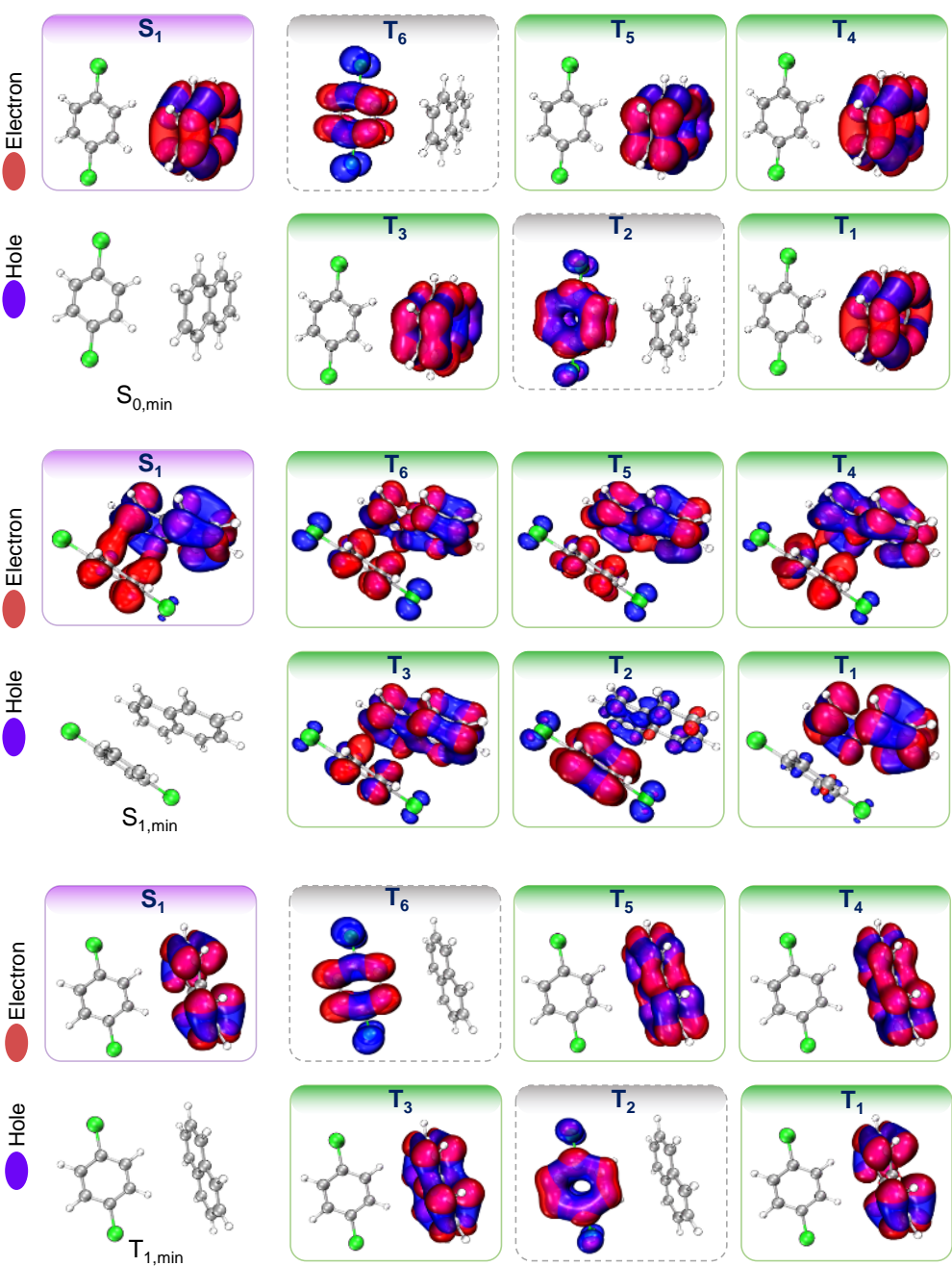

Figure 4. Energy levels of singlet $(\mathrm{S})$ and triplet $(\mathrm{T})$ states, spin-orbit coupling constants among $\mathrm{S}_{1}$ and $\mathrm{T}_{\mathrm{n}}$ states $(\mathrm{n}=1-6)$, electronic configuration calculated through electron-hole population of the combination of naphthalene (NL, guest) and 1,4-dichlorobenzene (DCB, host) at (a) the lowest ground state $\left(\mathrm{S}_{0, \mathrm{~min}}\right)$, (b) the lowest excited singlet state $\left(\mathrm{S}_{1, \mathrm{~min}}\right)$ and (c) the lowest triplet state $\left(\mathrm{T}_{1, \mathrm{~min}}\right)$. The triplet states for the effective intersystem crossing paths are marked by green color and the triplet states for ineffective paths are marked by grey color.

and almost no afterglow could be observed at 1 ppm by naked eyes. Consistently, the RTP band almost diminishes into baseline while fluorescence band $(\sim 300 \mathrm{~nm})$ of bulk DCB becomes clear for concentration of $10 \mathrm{ppm}$ and $1 \mathrm{ppm}$ in the steadystate PL spectra.

In short, the RTP intensity of NL/DCB was maximized with a proper doping concentration $(\sim 0.1 \%-1 \%)$ where the guest and host tend to be excited simultaneously. We thus reason NL and several DCB molecules in proximity interact with each other in the excited state to promote ISC of the system. To corroborate our claim, density functional theory (DFT) studies were carried out. Though mass ratio of $\sim 0.1 \%-1 \%$ are optimum for NL/DCB to emit efficient RTP, DFT simulation of NL in presence of several adjacent DCB molecules always lead to phase separation (Figure S8). Therefore, 1:1 of guest/host pair was selected to simulate electronic configuration of the exciton. As shown in Figure 4, we firstly optimized the ground state, excited singlet and triplet states of the 1:1 NL/DCB combo system and then obtained the energy levels, SOC constants and hole-electron population of each state. The calculated absorption, fluorescence and phosphorescence wavelengths well match the experimental values, which proves the reliability of the simulation model. At the minimum of $S_{0}$ state $\left(S_{0, m i n}\right)$, the triplet states from $\mathrm{T}_{1}$ to $\mathrm{T}_{6}$ are all below the $\mathrm{S}_{1}$ state, which provides potential channels for the ISC. The hole-electron population at $\mathrm{S}_{0, \min }$ indicate that $T_{1}, T_{3}, T_{4}$ and $T_{5}$ states share the same configuration with the $S_{1}$ states. Hence, these four triplet states will be the terminals for the effective ISC process. All the states at $S_{0, \min }$ only show the exciton population on either the guest NL molecule or the host DCB molecule, and the SOC values are also relatively low $\left(<0.1 \mathrm{~cm}^{-1}\right)$. When the exciton decays from the Frank-Condon point to the lowest excited singlet state $\left(S_{1, \min }\right)$, within around $+0.3 \mathrm{eV}$ above the $S_{1}$ state, there are $T_{1}$ to $T_{5}$ states being able to provide the ISC channels. Most importantly, at $S_{1, \min }$, all the calculated states show through-space hole-electron population between the host and guest molecule, which obviously demonstrates a cluster exciton character. The $S_{1}$ state and $T_{1}-T_{6}$ states show both through-space charge transfer and throughspace conjugation effect, which makes all the transitions from $\mathrm{S}_{1}$ to $\mathrm{T}_{1}-\mathrm{T}_{6}$ to be effective ISC paths. Meanwhile, the cluster exciton property at $S_{1, \min }$ endows the system with high SOC values to enhance the ISC process. After transition from $S_{1}$ state to the triplet state, the exciton finally populates on the NL molecule at the lowest triplet state $\left(\mathrm{T}_{1, \mathrm{~min}}\right)$ and produces the phosphorescence of NL molecule. Thus, the NL/DCB combo generates the cluster exciton at the $S_{1, \min }$ state, and it then leads to the highly efficient ISC process to generate sufficient triplet population. From the reduced density gradient analysis (Figure S9), we 
found that the through-space Van de Waals interactions could be the driving force for the formation of cluster excited state.

We also simulated the combination of NL/DMB and NL/DBB systems as shown in Figure S10 and S11. Due to the increased electron-donating ability and relatively bulky methoxyl groups of the DMB molecule, the distance between the NL and DMB molecule is further than the other two combinations and the exciton population shows more through-space charge transfer character. When decaying from Frank-Condon point to the $S_{1, \min }$, the number of cluster excited states increases $\left(S_{1}, T_{1}\right.$, $\mathrm{T}_{2}, \mathrm{~T}_{4}, \mathrm{~T}_{5}$ ) so that the effective ISC channels are enriched. However, lower steric proximity in NL/DMB combo largely elevates the triplet states. Despite of the cluster exciton character in the $\mathrm{T}_{4}$ and $\mathrm{T}_{5}$ states at the $\mathrm{S}_{1, \mathrm{~min}}$, the $\mathrm{T}_{4}$ and $\mathrm{T}_{5}$ states are $1 \mathrm{eV}$ higher than the $S_{1}$ state in energy, which makes them energetically unfavorable in the ISC process. Thus, we observed poor RTP performance in the NL/DMB system. The NL/DBB combo shows similar excited-state property as the NL/DCB combo with predominant cluster exciton property and abundant effective ISC channels at the $S_{1, \min }$ state. Due to the enhanced heavy atom effect, the SOC constants of NL/DCB are dramatically increased with the largest value of over $12 \mathrm{~cm}^{-1}$. Hence, the excessive ISC process makes the NL/DBB system show the shortest RTP lifetime. The lower RTP QY of NL/DBB than NL/DCB may result from heterogeneity and different radiationless paths in the guest/host material system. We then simulated the excited-state electronic configuration of isolated NL molecule for control. As shown in Figure S12, the NL molecule only shows similar exciton population in $S_{1}$ and $T_{1}$ states for all the three minima. Meanwhile, the NL molecule possesses negligible SOC constants $\left(<0.1 \mathrm{~cm}^{-1}\right)$ in the excited states. Thus, such inefficient ISC process resulted that no RTP can be detected in the pure NL system. The comparison between the pure NL system and the guest/host system further validated the dramatic strengthening effect of the cluster exciton on the RTP performance. Overall, the cluster exciton will lead to large SOC constants and increase the number of effective ISC channels to bridge the singlet with triplet states and finally lead to the enhanced RTP.

Application of the NL/DCB RTP. The super-efficient RTP of NL/DCB enables quick identification of NL molecules using bulk DCB as a "sensor" (an activator for NL RTP rather than a phosphor by itself). When little NL mothball was ground with excess amount of solid DCB, intense green RTP with duration of over $10 \mathrm{~s}$ emerged. Conversely, when mixing camphor (an aliphatic ketone with natural source) with DCB by grinding, no visible luminescence was noticed at all. Since the most ubiquitous pollution source of NL is vapor phase and the most common intake form for toxic NL is vapor inhalation due to its quick sublimation to the atmosphere at RT (no matter used as household mothballs or in chemical industry), we then tested the
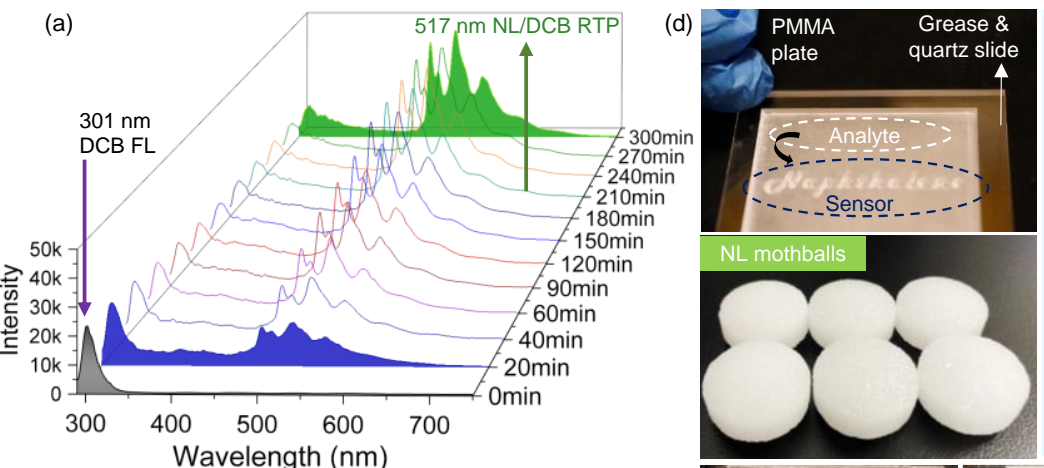

(e)

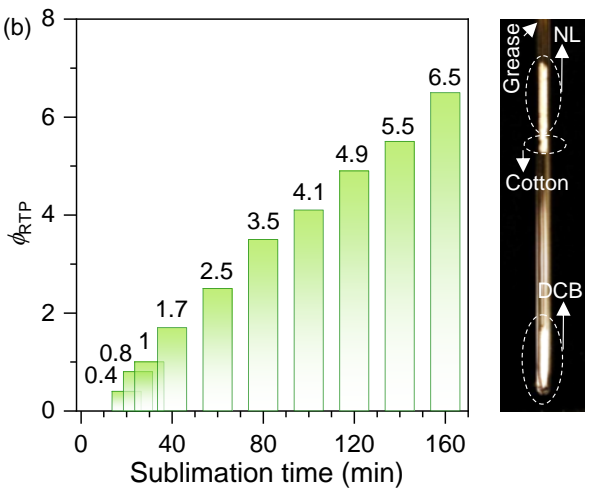

(c)

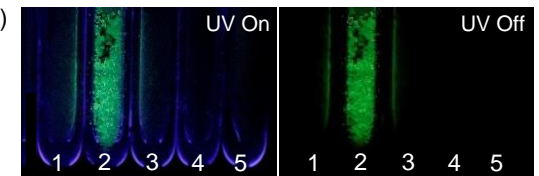

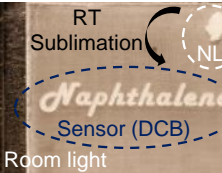
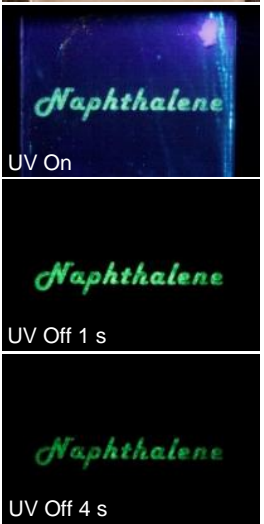
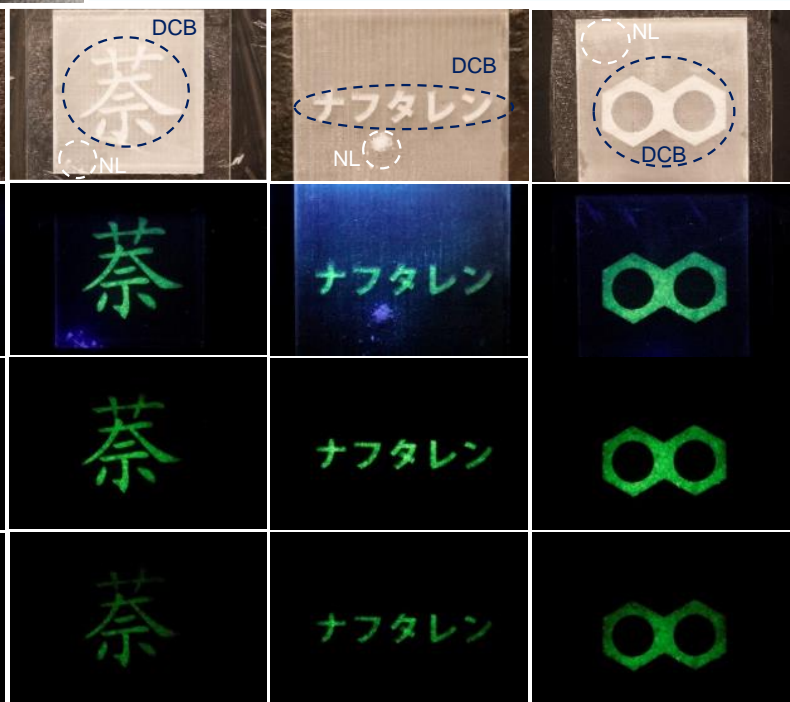

Figure 5. (a) Steady-state PL spectra of DCB sealed with non-contact NL solid in a quartz tube at RT for different times $\left(\lambda_{\mathrm{ex}}=280 \mathrm{~nm}\right)$. (b) Absolute RTP QY values over time monitored at DCB part sealed with non-contact NL solid in a quartz tube at RT $\left(\lambda_{\mathrm{ex}}=280 \mathrm{~nm}\right)$. The photo of DCB and NL solid separated by a piece of cotton in a sealed quartz tube which is used for the PL and QY measurements. (c) Photos of different hosts (1-DBM, 2-DCB, 3-TriCB, 4-TCB, 5-DBB) sealed with non-contact NL solid in a quartz tube at RT for $1 \mathrm{~h}$ under 254-nm UV irradiation and after UV was off. (d) Schematic illustration of sensing device (left) and process (right) for NL mothball vapor using "turn-on" RTP when bulk DCB in the shape of characters meets NL vapor in sealed PMMA plate. (e) RTP and afterglow photos of the DCB sensor part after exposure to small amount of non-contact NL solid for $2 \mathrm{~h}$ in a sealed PMMA plate $\left(\lambda_{\mathrm{UV}}=254 \mathrm{~nm}\right)$. 
ability of DCB to detect NL vapor based on "turn-on" type RTP. When almost non-luminescent solid DCB was sealed with NL solid in a quartz tube without direct contact, the RTP intensity monitored at the DCB part kept increasing as shown by the phosphorescence band $\left(\lambda_{\mathrm{em}-\max }=517 \mathrm{~nm}\right.$ with vibrational peaks at $481,517,559$, and $605 \mathrm{~nm}$ ) in the steady-state PL spectra as well as RTP QY values measured over a period of several hours (Figure 5a-b). This is caused by rapid and continuous sublimation of NL molecules into the DCB solid at RT. Moreover, only DCB among the five studied hosts could give rise to notable green RTP when using the same non-contact sublimation method within a timescale of $1 \mathrm{~h}$ (Figure 5c). Finally, a small PMMA device for NL vapor detection was fabricated (Figure 5d). Inside the groove of PMMA plate, the sculptured character or pattern part (English, Chinese, Japanese and chemical structure version of naphthalene, respectively) is filled with DCB solid. Then, little amount of NL solid was placed on the other part of the groove, staying apart from DCB solid, and sealed with quartz slide subsequently at RT. Within just one minute, the character or pattern (sensor) part could show visible green afterglow to naked eyes, indicating the sublimation of NL molecules into DCB solid is extremely fast at RT. Within $2 \mathrm{~h}$, noticeable steady-state green RTP with seconds-long afterglow was observed (Figure 5e). These results demonstrated that DCB is a specific and sensitive RTP "turn-on" sensor for both solidstate and gaseous NL based on intuitive afterglow phenomenon at ambient conditions.

\section{CONCLUSION}

In this work, we disclosed a neglected RTP phenomenon from a simple guest/host system with commercial sources. Particularly, the guest (NL) is a traditional and banned component for widespread mothballs while the host (DCB) is a safer alternative to NL for manufacturing mothballs. The efficiency of the green RTP surpasses $20 \%$ ( $\lambda_{\mathrm{ex}}=260$ or $290 \mathrm{~nm}$ ) with simultaneously ultralong lifetime, a performance better than that of the vast majority of metal-free RTP materials reported to date. In addition to the role halogens played, the strong RTP of the unique NL/DCB system was proposed to mainly arise from a cluster exciton where electron and hole are delocalized (spanning the guest and the host) for promoting ISC. The synergistic interaction between the guest and host in the transient excited state is different from conventional energy transfer model where only one species, either the guest or the host, could be in the excited state at any time. This interpretation based on detailed experimental and theoretical evidence will advance the understanding in purely organic RTP systems. On the other hand, since naked eyes are most sensitive to green light, the RTP of NL/DCB with QY of $\sim 20 \%$ is indeed quite recognizable by common people, even under soft room lighting. It was demonstrated that the initial "dark" bulk DCB solid could show notable green afterglow upon mixing with trace amount of NL solid or vapor. In addition to abused globally as moth repellents, NL is also a major product of coal tar and a precursor for preparing other aromatic compounds. Furthermore, NL is a characteristic substance found in certain interstellar medium ${ }^{58-59}$ which can indicate the evolution of galaxies, and also a compound synthesized by some fungi ${ }^{60}$ to protect their host plants. Therefore, the bright RTP of the unique NL/DCB system is of vital significance for sensing applications, not only in identifying illegal mothballs, but also in fields of chemical industry, astronomy and biosciences.

\section{Supporting Information}

Materials, methods, supplementary photophysical properties at RT and $77 \mathrm{~K}$ and DFT data, including Figures S1-S12.

\section{AUTHOR INFORMATION}

\section{Corresponding Author}

*tangbenz@cuhk.edu.cn

\section{Author Contributions}

॥X. Z., J. L. and B. C. contributed equally.

\section{Notes}

The authors declare no competing financial interest.

\section{ACKNOWLEDGMENT}

This work was financially supported by the National Natural Science Foundation of China (21788102), the Research Grants Council of Hong Kong (16305518, 16307020, C6014-20W, C6009-17G, and N_HKUST609/19), the Innovation and Technology Commission (ITC-CNERC14SC01), and the Natural Science Foundation of Guangdong Province (2019B121205002 and 2019B030301003). X. Z. thank Prof. Jian Ping Gong at Hokkaido University for her kind help in fabricating PMMA plates used in Figure 5.

\section{REFERENCES}

(1) Batterman, S.; Chin, J. Y.; Jia, C.; Godwin, C.; Parker, E.; Robins, T.; Max, P.; Lewis, T., Indoor Air 2012, 22 (4), 266278.

(2) In Comprehensive Organic Name Reactions and Reagents, pp 1227-1229.

(3) Todisco, V.; Lamour, J.; Finbero, L., N. Engl. J. Med. 1991, 325 (23), 1660-1661.

(4) Rahman, M. M.; Mowla, S. M.; Rahim, A.; Chowdhury, F. R.; Jahan, S.; Hasan, M. N., J Coll Physicians Surg Pak 2012, 22 (11), 740-741.

(5) Kundra, T. S.; Bhutatani, V.; Gupta, R.; Kaur, P., Journal of Clinical and Diagnostic Research: JCDR 2015, 9 (8), UD01.

(6) Memar, H.; Evini, P. E. T.; Seirafi, G.; Rahimi, M., 2021.

(7) Sanctucci, K.; Shah, B., Acad. Emerg. Med. 2000, 7 (1), $42-$ 47.

(8) Lincoln, D. E.; Lawrence, B. M., Phytochemistry 1984, 23 (4), 933-934.

(9) Lewis, G. N.; Kasha, M., J. Am. Chem. Soc. 1944, 66 (12), 2100-2116.

(10) El-Sayed, M. A., Acc. Chem. Res. 1971, 4 (1), 23-31.

(11) Zhao, W.; He, Z.; Tang, B. Z., Nat. Rev. Mater. 2020, 5 (12), 869-885.

(12) Gao, R.; Kodaimati, M. S.; Yan, D., Chem. Soc. Rev. 2021.

(13) Kabe, R.; Adachi, C., Nature 2017, 550 (7676), 384.

(14) Chen, C.; Chi, Z.; Chong, K. C.; Batsanov, A. S.; Yang, Z.; Mao, Z.; Yang, Z.; Liu, B., Nat. Mater. 2021, 20 (2), 175-180.

(15) Ding, B.; Ma, L.; Huang, Z.; Ma, X.; Tian, H., Sci. Adv. 2021, 7 (19), eabf9668.

(16) Jinnai, K.; Kabe, R.; Lin, Z.; Adachi, C., Nat. Mater. 2021, $1-7$.

(17) Alam, P.; Cheung, T. S.; Leung, N. L.; Zhang, J.; Guo, J.; Du, L.; Kwok, R. T.; Lam, J. W.; Zeng, Z.; Phillips, D. L., J. Am. Chem. Soc. 2022.

(18) Wang, J.; Gu, X.; Ma, H.; Peng, Q.; Huang, X.; Zheng, X.; Sung, S. H.; Shan, G.; Lam, J. W.; Shuai, Z., Nat. Commun. 2018, 9 (1), 2963. 
(19) Wang, T.; Su, X.; Zhang, X.; Nie, X.; Huang, L.; Zhang, X.; Sun, X.; Luo, Y.; Zhang, G., Adv. Mater. 2019, 31 (51), 1904273.

(20) Wei, J.; Liang, B.; Duan, R.; Cheng, Z.; Li, C.; Zhou, T.; Yi, Y.; Wang, Y., Angew. Chem. Int. Ed. 2016, 55 (50), 1558915593.

(21) Zhao, W.; He, Z.; Lam, J. W.; Peng, Q.; Ma, H.; Shuai, Z.; Bai, G.; Hao, J.; Tang, B. Z., Chem 2016, 1 (4), 592-602.

(22) Jiang, K.; Wang, Y.; Gao, X.; Cai, C.; Lin, H., Angew. Chem. Int. Ed. 2018, 57 (21), 6216-6220.

(23) Li, Q.; Zhou, M.; Yang, M.; Yang, Q.; Zhang, Z.; Shi, J., Nat. Commun. 2018, 9 (1), 734.

(24) Ma, H.; Peng, Q.; An, Z.; Huang, W.; Shuai, Z., J. Am.Chem.Soc. 2018, 141 (2), 1010-1015.

(25) Su, Y.; Phua, S. Z. F.; Li, Y.; Zhou, X.; Jana, D.; Liu, G.; Lim, W. Q.; Ong, W. K.; Yang, C.; Zhao, Y., Sci. Adv. 2018, 4 (5), eaas9732.

(26) Tao, S.; Lu, S.; Geng, Y.; Zhu, S.; Redfern, S. A.; Song, Y.; Feng, T.; Xu, W.; Yang, B., Angew. Chem. Int. Ed. 2018, 57 (9), 2393-2398

(27) Yang, J.; Zhen, X.; Wang, B.; Gao, X.; Ren, Z.; Wang, J.; Xie, Y.; Li, J.; Peng, Q.; Pu, K., Nat. Commun. 2018, 9 (1), 840.

(28) Gu, L.; Shi, H.; Bian, L.; Gu, M.; Ling, K.; Wang, X.; Ma, H.; Cai, S.; Ning, W.; Fu, L., Nat. Photonics 2019, 1.

(29) Lei, Y.; Dai, W.; Guan, J.; Guo, S.; Ren, F.; Zhou, Y.; Shi, J.; Tong, B.; Cai, Z.; Zheng, J., Angew. Chem. Int. Ed. 2020, 59 (37), 16054-16060.

(30) Ye, W.; Ma, H.; Shi, H.; Wang, H.; Lv, A.; Bian, L.; Zhang, M.; Ma, C.; Ling, K.; Gu, M., Nat. Mater. 2021, 20 (11), 15391544.

(31) Zhang, Y.; Su, Y.; Wu, H.; Wang, Z.; Wang, C.; Zheng, Y.; Zheng, X.; Gao, L.; Zhou, Q.; Yang, Y., J. Am. Chem. Soc. 2021, 143 (34), 13675-13685.

(32) Wang, Y.; Yang, J.; Fang, M.; Yu, Y.; Zou, B.; Wang, L.; Tian, Y.; Cheng, J.; Tang, B. Z.; Li, Z., Matter 2020, 3 (2), 449463

(33) Zhang, G.; Palmer, G. M.; Dewhirst, M. W.; Fraser, C. L., Nat. Mater. 2009, 8 (9), 747.

(34) Chen, X.; Xu, C.; Wang, T.; Zhou, C.; Du, J.; Wang, Z.; Xu, H.; Xie, T.; Bi, G.; Jiang, J., Angew. Chem. Int. Ed. 2016, 55 (34), 9872-9876.

(35) Zhen, X.; Tao, Y.; An, Z.; Chen, P.; Xu, C.; Chen, R.; Huang, W.; Pu, K., Adv. Mater. 2017, 29 (33), 1606665.

(36) Li, D.; Lu, F.; Wang, J.; Hu, W.; Cao, X.-M.; Ma, X.; Tian, H., J. Am. Chem. Soc. 2018, 140 (5), 1916-1923.

(37) He, Z.; Gao, H.; Zhang, S.; Zheng, S.; Wang, Y.; Zhao, Z.; Ding, D.; Yang, B.; Zhang, Y.; Yuan, W. Z., Adv. Mater. 2019, 1807222

(38) Wang, B.; Yu, Y.; Zhang, H.; Xuan, Y.; Chen, G.; Ma, W.; Li, J.; Yu, J., Angew. Chem. 2019, 131 (51), 18614-18619.

(39) Yu, X.; Zhang, H.; Yu, J., Aggregate 2021, 2 (1), 20-34.

(40) Bolton, O.; Lee, K.; Kim, H.-J.; Lin, K. Y.; Kim, J., Nat. Chem. 2011, 3 (3), 205.

(41) Bian, L.; Shi, H.; Wang, X.; Ling, K.; Ma, H.; Li, M.; Cheng, Z.; Ma, C.; Cai, S.; Wu, Q., J. Am. Chem. Soc. 2018, 140 (34), 10734-10739.

(42) Tang, S.; Yang, T.; Zhao, Z.; Zhu, T.; Zhang, Q.; Hou, W.; Yuan, W. Z., Chem. Soc. Rev. 2021.

(43) Hutchison Jr, C. A.; Mangum, B. W., J. Chem. Phys. 1961, 34 (3), 908-922.

(44) Marchetti, A. P.; Kearns, D. R., J. Am.Chem.Soc. 1967, 89 (4), 768-777.

(45) Klymko, P.; Kopelman, R., J. Phys. Chem. 1982, 86 (19), 3686-3688.
(46) Diaz Garcia, M. E.; Sanz-Medel, A., Anal. Chem. 1986, 58 (7), 1436-1440.

(47) Mei, J.; Leung, N. L.; Kwok, R. T.; Lam, J. W.; Tang, B. Z., Chem. Rev. 2015, 115 (21), 11718-11940.

(48) Nie, X.; Huang, W.; Zhou, D.; Wang, T.; Wang, X.; Chen, B.; Zhang, X.; Zhang, G., Aggregate 2022, e165.

(49) Yang, J.; Fang, M.; Li, Z., Aggregate 2020, 1 (1), 6-18.

(50) McClure, D. S., J. Chem. Phys. 1949, 17 (7), 665-666.

(51) Kasha, M., J. Chem. Phys. 1952, 20 (1), 71-74.

(52) Zhang, X.; Du, L.; Zhao, W.; Zhao, Z.; Xiong, Y.; He, X.; Gao, P. F.; Alam, P.; Wang, C.; Li, Z., Nat. Commun. 2019, 10 (1), 1-10.

(53) Engel, G. S.; Calhoun, T. R.; Read, E. L.; Ahn, T.-K.; Mančal, T.; Cheng, Y.-C.; Blankenship, R. E.; Fleming, G. R., Nature 2007, 446 (7137), 782.

(54) Collini, E.; Wong, C. Y.; Wilk, K. E.; Curmi, P. M.; Brumer, P.; Scholes, G. D., Nature 2010, 463 (7281), 644.

(55) Panitchayangkoon, G.; Hayes, D.; Fransted, K. A.; Caram, J. R.; Harel, E.; Wen, J.; Blankenship, R. E.; Engel, G. S., Proc. Natl. Acad. Sci. USA 2010, 107 (29), 12766-12770.

(56) Chenu, A.; Scholes, G. D., Annu. Rev. Phys. Chem. 2015, $66,69-96$.

(57) Giachino, G. G.; Kearns, D. R., J. Chem. Phys. 1970, 52 (6), 2964-2974

(58) Tielens, A. G., Annu. Rev. Astron. Astrophys. 2008, 46, 289-337.

(59) Parker, D. S.; Zhang, F.; Kim, Y. S.; Kaiser, R. I.; Landera, A.; Kislov, V. V.; Mebel, A. M.; Tielens, A., Proc. Natl. Acad. Sci. USA 2012, 109 (1), 53-58.

(60) Daisy, B. H.; Strobel, G. A.; Castillo, U.; Ezra, D.; Sears, J.; Weaver, D. K.; Runyon, J. B., Microbiology 2002, 148 (11), 3737-3741. 
ToC

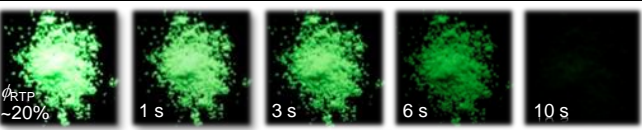

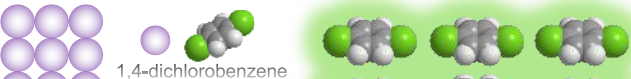

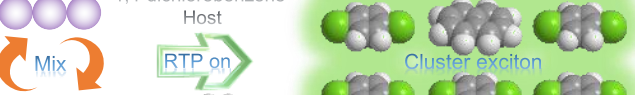

088 10 dio do

Naphthalene
cumet 